\title{
Les sites des Services Communs de Documentation en France : contenus et perspectives
}

The Sites of the Services Communs de Documentation in

France: Their Contents and Outlook

Sitios servicios de documentación públicos en Francia: contenidos y perspectivas

\section{Lylette Lacôte-Gabrysiak}

Volume 54, numéro 4, octobre-décembre 2008

URI : https://id.erudit.org/iderudit/1029189ar

DOI : https://doi.org/10.7202/1029189ar

Aller au sommaire du numéro

Éditeur(s)

Association pour l'avancement des sciences et des techniques de la documentation (ASTED)

ISSN

0315-2340 (imprimé)

2291-8949 (numérique)

Découvrir la revue

Citer cet article

Lacôte-Gabrysiak, L. (2008). Les sites des Services Communs de Documentation en France : contenus et perspectives. Documentation et bibliothèques, 54(4),

265-272. https://doi.org/10.7202/1029189ar
Résumé de l'article

Cet article présente les résultats de l'examen des sites de Services Communs de Documentation (SCD) français, réalisé entre novembre 2007 et mars 2008. Après une présentation de la localisation de ces sites et des différents types de services qu'ils proposent, les résultats d'une enquête sur l'usage du site du SCD de l'Université de Nancy 2 par des étudiants sont analysés. Enfin, un éclairage fondé sur une vision des sites de SCD en tant qu'outil de communication et une revue des difficultés et contraintes actuelles sont présentés.
Tous droits réservés ( $)$ Association pour l'avancement des sciences et des techniques de la documentation (ASTED), 2008
Ce document est protégé par la loi sur le droit d'auteur. L'utilisation des services d'Érudit (y compris la reproduction) est assujettie à sa politique d'utilisation que vous pouvez consulter en ligne.

https://apropos.erudit.org/fr/usagers/politique-dutilisation/ 


\title{
Les sites des Services Communs de Documentation en France : contenus et perspectives
}

\author{
LYLETTE LACÔTE-GABRYSIAK \\ IUT Nancy-Charlemange, Université Nancy 2 \\ Chercheure associée à l'équipe Site du Loria \\ lylette.lacote@univ-nancy2.fr
}

\section{RÉSUMÉ | ABSTRACTS | RESUMEN}

Cet article présente les résultats de l'examen des sites de Services Communs de Documentation (SCD) français, réalisé entre novembre 2007 et mars 2008. Après une présentation de la localisation de ces sites et des différents types de services qu'ils proposent, les résultats d'une enquête sur l'usage du site du SCD de l'Université de Nancy 2 par des étudiants sont analysés. Enfin, un éclairage fondé sur une vision des sites de SCD en tant qu'outil de communication et une revue des difficultés et contraintes actuelles sont présentés.

\section{The Sites of the Services Communs de Documentation in} France: Their Contents and Outlook

This article presents the results of a survey of the sites of the Services Communs de Documentation (SCD) in France; the survey was undertaken between November 2007 and March 2008. After identifying the locations and the services offered by the SCDs, the author analyses the use made by students of the SCD at the Universite de Nancy 2. The author concludes the article with her perspective of a long-term vision for the SCD sites as a communication service, as well as some of the challenges and constraints.

Sitios servicios de documentación públicos en Francia : contenidos y perspectivas

Este artículo presenta las conclusiones sobre la evaluación de los sitios de servicios de documentación públicos ( $S C D$, por sus siglas en francés), realizada entre noviembre de 2007 y marzo de 2008. Luego de una presentación de la ubicación de los sitios y de los diferentes servicios que ofrecen, se analizaron los resultados de una encuesta sobre el uso que los estudiantes hacen del sitio SCD de la Universidad de Nancy 2. Por último, se incluye una opinión basada en una perspectiva de los sitios SCD como herramienta de comunicación, y un análisis de las dificultades y limitaciones actuales.
E N AVANT-PROPOS, il nous paraît utile de présenter succinctement le fonctionnement de la documentation universitaire en France. Les Services Communs de Documentation (SCD), dont il est question dans le présent article, ont été créés par décret en 1985 afin de mettre en œuvre la politique documentaire des différentes bibliothèques de l'université (BUs) : les bibliothèques universitaires, mais également celles rattachées aux différents laboratoires de recherche, aux instituts ou aux unités de formation. Auparavant, chaque BU avait sa propre politique documentaire et gérait son budget, son personnel et ses locaux de manière indépendante. Rappelons que les BUs sont rattachées au ministère de l'Éducation Nationale, que leur financement est national et leur personnel constitué par des fonctionnaires d'État accédant à leur fonction à la suite de concours nationaux. Quant aux autres bibliothèques dans l'université, elles étaient autonomes tant au niveau de leur création, de leur financement et de l'ensemble de leur gestion. Les SCD permettent donc de cumuler les moyens et de mettre en application une politique globale de documentation et de gestion (budget, locaux, personnel) dans chaque université. Si la création des SCD est possible depuis 1985, elle n'est pas obligatoire et chaque université a décidé de la création de son SCD quand elle le jugeait bon. À l'heure actuelle, presque toutes les universités (à l'exception de quelques écoles) ont créé leur SCD. La loi de 2007 sur l'autonomie des universités donne plus de prérogatives aux présidents d'université sur la gestion des SCD. Comme ces structures dépendent d'une université, elles présentent certaines caractéristiques liées à celle-ci. En effet, certaines universités sont pluridisciplinaires alors que d'autres sont spécialisées, certaines assurent uniquement des cursus assez généraux alors que d'autres intègrent des cursus professionnels beaucoup plus spécialisés. De même, certaines universités sont importantes en termes de taille, et donc de moyens, d'autres sont beaucoup plus modestes. Outre l'acquisition et la mise à disposition des documents et des informations, la fonction des SCD est également pédagogique. Il est à noter que les SCD en France sont d'abord au service de la communauté universitaire et servent une clientèle extérieure très restreinte $(9 \%$ selon l'Association des 
bibliothécaires de France 2007, p. 93) même si l'accueil de ce public est tout à fait possible. Il y a également peu de liens avec l'environnement local et régional ainsi qu'avec les autres types de bibliothèques, notamment les bibliothèques municipales ${ }^{1}$.

Le SCD de l'Université de Nancy 2 regroupe trois bibliothèques universitaires liées à trois sites différents d'enseignement et donc aux spécialités afférentes: les BUs de Lettres (littérature, langues, sciences humaines), de Droit/Sciences Economiques et la BIUT (bibliothèque d'un centre de formation professionnelle regroupant des étudiants de la $1^{\mathrm{e}}$ à la $3^{\mathrm{e}}$ année, dans les domaines de l'informatique, de l'information/communication, des techniques de commercialisation, de la gestion des entreprises et des administrations ainsi que des services et réseaux de communication). À ces bibliothèques s'ajoutent 32 bibliothèques spécialisées liées à des laboratoires de recherche ou à des instituts. Le SCD de l'Université Henri Poincaré de Nancy 1, dont il sera également question par la suite, regroupe les BUs de sciences, de médecine, de pharmacie ainsi que de nombreuses autres bibliothèques de recherche ou d'institut liés aux sciences exactes et médicales.

La documentation universitaire a beaucoup évolué au cours de la dernière décennie. La multiplication des documents numériques, notamment à travers lémergence des périodiques électroniques, a modifié non seulement les politiques d'acquisition, mais également le rapport aux usagers qui sest, en partie du moins, dématérialisé. La mise en place des SCD permet aujourd'hui d'accéder à une part importante (suffisante dans de nombreux cas) de la documentation sans avoir à se rendre physiquement à la bibliothèque. Bien sûr, des contraintes existent : certains documents peuvent nêtre accessibles que depuis les postes informatiques de l'université ; néanmoins, on peut considérer, aujourd'hui, que les sites de bibliothèques sont davantage qu'un simple reflet des lieux physiques. Cest pourquoi nous avons voulu avoir une idée de ce qui est présenté sur les sites des $\mathrm{SCD}^{2}$. Dans un second temps, nous avons voulu envisager ces sites sous un angle assez inhabituel dans un contexte bibliothéconomique en les considérant comme des outils de communication, ceci afin de poser un nouvel éclairage sur leur contenu et leur fonctionnement.

\section{Les sites des SCD aujourd'hui}

Pour établir la liste des sites de SCD à examiner, nous avons consulté le site du ministère français de l'Éducation3. Ce site recense 90 adresses de SCD ou de

1. Pour une liste des coordonnées des différents $S C D$, ainsi qu'un accès aux statistiques sur l'ensemble de leurs activités, on peut consulter le site de l'Enseignement supérieur et de la recherche, sous-direction des bibliothèques et de l'information scientifique : http://www.sup.adc.education.fr/bib/

2. Merci à l'équipe d'étudiants de Métiers de livre de l'IUT Nancy-Charlemagne qui a travaillé avec moi sur ce projet.

3. www.sup.adc.education.fr/bib/

\section{Tableau 1.}

Spécialités des SCD en France

\begin{tabular}{|c|c|c|}
\hline SPÉCIALITÉ & NOMBRE CITÉ & FréQQuence \\
\hline Shs Lettres & 33 & $36,70 \%$ \\
\hline Droit Sc-éco gestion & 29 & $32,20 \%$ \\
\hline Sc méd pharma & 25 & $27,80 \%$ \\
\hline Toutes spécialités & 42 & $46,70 \%$ \\
\hline Autres & 11 & $12,20 \%$ \\
\hline Total observé & 90 & \\
\hline
\end{tabular}

Total observé

90

\section{Tableau 2.}

Informations et liens présents sur la page d'accueil des sites de SCD

\begin{tabular}{|c|c|c|}
\hline Page d'accuell & NOMBRE CITÉ & FréQuence \\
\hline Non réponse & 1 & $1,10 \%$ \\
\hline Lien vers le site de l'université & 72 & $80 \%$ \\
\hline Présentation du SCD & 52 & $57,80 \%$ \\
\hline Catalogue en ligne & 78 & $86,70 \%$ \\
\hline Bases de données & 56 & $62,20 \%$ \\
\hline Périodiques électroniques & 64 & $71,10 \%$ \\
\hline Thèses en ligne & 27 & $30 \%$ \\
\hline $\begin{array}{l}\text { Cours en ligne/annales } \\
.\end{array}$ & 12 & $13,30 \%$ \\
\hline Contacts & 62 & $68,90 \%$ \\
\hline Aide en ligne & 27 & $30 \%$ \\
\hline Total observé & 90 & \\
\hline
\end{tabular}

services communs inter-établissement de documentation. Comme le révèle le Tableau 1, les SCD peuvent être spécialisés ou non et appartenir à différentes disciplines.

Il nous est apparu intéressant de déterminer, en nous servant des adresses relevées sur le site du ministère, combien de ces sites étaient des sites autonomes et combien étaient inclus dans le site plus vaste de leur université de rattachement ; 65 sites sur 90, soit $72,2 \%$, sont indépendants, alors que 27 sites, soit $30 \%$, sont accessibles sur le site de l'université (il y avait une nonréponse). Cette donnée ne peut être considérée comme forcément exhaustive puisqu'il peut exister à la fois un site SCD indépendant et une page sur le site de l'université. Dans cet article, le contenu des pages d'accueil et de l'ensemble des sites fera référence soit au site du SCD lui-même, soit au site de l'université dans le cas où le SCD n'a pas de site particulier. Par exemple, l'adresse donnée pour le SCD de Nancy 2 est la suivante : http:// univ-nancy2.fr/SCD/. Or, il existe également un portail documentaire d'interrogation du catalogue : http://scd. univ-nancy2.fr/ qui donne accès aux bases de données, revues électroniques et thèses en ligne4. Étant donné la méthodologie employée, il ne nous est pas possible de

4. Je voudrais remercier pour leur collaboration et leur patience Anne-Marie Thummel, Annabelle Clausse et, particulièrement, Catherine Benedic conserva- 


\section{Tableau 3.}

Informations concernant le $S C D$ présentes sur les sites des SCD

\begin{tabular}{|c|c|c|}
\hline INFO SUR SCD & Nв. Cir. & FrééQ. \\
\hline Non réponse & 6 & $6,70 \%$ \\
\hline Coordonnées des différentes BU & 73 & $81,10 \%$ \\
\hline Présentations des différentes $\mathrm{BU}$ & 67 & $74,40 \%$ \\
\hline Visites virtuelles des différentes $B U$ & 16 & $17,80 \%$ \\
\hline Description du fonctionnement & 42 & $46,70 \%$ \\
\hline Règlement & 51 & $56,70 \%$ \\
\hline Plan d'accès des différentes BU & 45 & $50 \%$ \\
\hline $\begin{array}{l}\text { Horaires des différentes BU } \\
\text {. }\end{array}$ & 67 & $74,40 \%$ \\
\hline $\begin{array}{l}\text { Historique, fonds anciens } \\
\text {. }\end{array}$ & 10 & $11,10 \%$ \\
\hline Actu du SCD & 50 & $55,60 \%$ \\
\hline Aide en ligne & 33 & $36,70 \%$ \\
\hline Total observé & 90 & \\
\hline
\end{tabular}

dire si d'autres SCD sont dans la même situation. Il faut surtout tenir compte de l'importance des ENT au cours des dernières années. Les ENT, ou Espace Numérique de Travail, sont les intranets des universités sur lesquels les étudiants peuvent accéder à leurs résultats, leurs emplois du temps, etc. ainsi qu’à une section documentaire gérée par le SCD. Ces ENT impliquent une nouvelle implantation des sites des SCD souvent présents également sur ce support (Bourrion, 2007). Cela ne signifie pas l'abandon du site Web lui-même, mais peut entraîner une nouvelle répartition des ressources. C'est également le cas à l'Université de Nancy 2, où le SCD est présent sur l'ENT (sous un onglet Documentation) et offre, par ce biais, accès à son catalogue, aux bases de données et aux thèses en ligne. Cette possibilité d'accès multiple aux ressources peut s'avérer relativement déstabilisante pour les usagers.

Nous avons ensuite voulu savoir si les dates de création et de mise à jour des sites étaient mentionnées. La date de création des sites nétait mentionnée que dans $10 \%$ des cas et la date de la dernière mise à jour dans $23,3 \%$ des cas. C'est assez peu. La date de la dernière mise à jour notamment, qui peut différer en fonction des rubriques, est une donnée intéressante pour les usagers d'un site, car elle leur permet d'avoir une idée de l'actualité des données présentées.

Nous avons ensuite essayé de voir quels étaient les éléments mentionnés sur la page d’accueil. Cette information semblait importante dans une logique d'ergonomie des sites. Il semble fondamental que loffre d'un site soit claire dès la première page. Il est également pratique de pouvoir accéder rapidement aux services recherchés. Les pages d'accueil contiennent des liens et des informations de différentes natures, présentés au Tableau 2.
On voit sur le Tableau 2 que le rattachement institutionnel des SCD est bien pris en compte puisque $80 \%$ des sites renvoient au site de l'université. Dans le cas où le SCD serait présenté sur le site de l'université, cela correspond à la possibilité d’aller directement sur la page d'accueil de l'université. Les SCD sont des services de l'université, il paraît donc normal que ce lien existe. Le lien vers le catalogue est le service le plus présent dès la page d'accueil sur $86,7 \%$ des sites SCD. Ce service a été généralement le premier à être mis en place sur les sites SCD, dès que ceux-ci ont pu être autre chose qu'une simple présentation "vitrine ». Laccès direct à la documentation numérique est possible sur presque tous les sites dès la première page. C'est donc bien une tendance affirmée: les pages d'accueil sont riches et complètes, elles permettent un accès direct aux différents services offerts.

Afin de mieux cerner le contenu des sites SCD, nous avons décomposé celui-ci en différentes catégories. Il nous paraissait assez logique de partir de l'hypothèse que les sites des SCD contiendraient vraisemblablement :

- des informations, pratiques notamment, sur le SCD lui-même et sur les différentes BUs qui le composent ;

- le catalogue en ligne avec plus ou moins de fonctionnalités disponibles;

- des ressources électroniques, disponibles, au moins pour une partie d'entres elles, sur un intranet ;

- d'autres services en ligne (signets, demande de PEB, service de questions/réponses, didacticiels, etc.).

Concernant les informations sur le SCD lui-même nous aboutissons aux observations suivantes, présentées au Tableau 3.

Notons que l'aide en ligne dont il est question au Tableau 3 correspond à une aide dirigée vers l'utilisation du SCD lui-même, et non pas à un service de question/ réponses de type Biblioses@me5 ou Guichet du savoir6, ce type de service ayant été recensé plutôt au Tableau 4.

Nous pouvons constater que les SCD, sur leur site en tout cas, ne donnent pas toujours facilement accès à certaines des informations pratiques que l'on pourrait s'attendre à y trouver (les horaires douverture des BUs, par exemple, n'y sont présents que dans $67 \%$ des cas). Il semble également que la présentation des bibliothèques physiques, du règlement et des fonds soit peu présente sur ces sites. Cela s'explique par le fait que les étudiants sont informés par d’autres biais (visites, règlements distribués et/ou affichés, etc.). Cela va dans le sens d'un contenu des sites davantage orienté vers la fourniture de services en ligne que vers une vitrine des bibliothèques, ce qui parait logique aujourd'hui.

5. Biblioses@me est un service de questions/réponses à distance coopératif qui regroupe 14 bibliothèques françaises. Le grand public peut poser aux bibliothécaires des questions sur tous les sujets par mail, téléphone ou chat.

6. Les Guichets du savoir est le service de questions/réponses en ligne mis en place par la Bibliothèque municipale de Lyon. 


\section{Tableau 4.}

Fonctions des catalogues accessibles par les SCD

\begin{tabular}{|c|c|c|}
\hline Catalogue en ligne & NOMBRE crté́ & FréQuence \\
\hline Non réponse & 11 & $12,20 \%$ \\
\hline $\begin{array}{l}\text { Interrogation de l'ens. ou } \\
\text { choix du cata. d'un ou } \\
\text { plusieurs BU }\end{array}$ & 65 & $72,20 \%$ \\
\hline $\begin{array}{l}\text { Réservation possible } \\
\text { en ligne }\end{array}$ & 24 & $26,70 \%$ \\
\hline Panier & 66 & $73,30 \%$ \\
\hline Tri par pertinence & 25 & $27,80 \%$ \\
\hline $\begin{array}{l}\text { Recherche simultanée } \\
\text { sur plusieurs type de } \\
\text { fonds }\end{array}$ & 63 & $70,00 \%$ \\
\hline $\begin{array}{l}\text { Accès direct aux docs } \\
\text { électroniques }\end{array}$ & 31 & $34,40 \%$ \\
\hline Total observé & 90 & \\
\hline
\end{tabular}

Les sites des SCD proposent systématiquement laccès au catalogue en ligne. À ce niveau, différentes fonctions sont offertes (Tableau 4).

Les non-réponses correspondent à des catalogues qui nétaient pas accessibles le jour où à été effectué le dépouillement.

Dans le Tableau 4, on voit bien que certaines des fonctions maintenant développées dans les OPACs (catalogues en ligne) ne sont pas encore très courantes, comme la possibilité de réserver en ligne ou le choix $\mathrm{du}$ tri par pertinence. En revanche, l'interrogation possible sur un ou plusieurs catalogues ainsi que la mise à disposition d'un panier sont souvent proposés (dans plus de $80 \%$ des cas, si on ne tient pas compte des 11 non-réponses).

La fourniture de documents numériques sur les sites des SCD est un service attendu. Il existe différents types de documents numériques (Tableau 5 ) :

Concernant ces résultats, il faut tenir compte de certaines ambiguïtés: les ressources payantes ne sont accessibles que depuis les intranets locaux auxquels nous ne pouvions accéder. Il peut sembler étonnant que lon trouve plus de périodiques électroniques que de bases de données dans loffre de documents numériques, mais il faut bien penser qu'il s'agit de l'offre en ligne, sur le site des SCD. Rien ne nous permet de savoir ce qui se trouve sur lintranet ou sur les ENT. Il en va de même de l'offre d'usuels (encyclopédies, dictionnaires, répertoires). Des usuels électroniques doivent être présents dans l'ensemble des BUs françaises, mais un lien n'y conduit pas forcément depuis leur site Web. Certains sites renvoient ainsi vers l'Encyclopaedia Universalis (connexion qui, bien sûr, se heurte rapidement à une demande de mot de passe), mais d'autres sites ne mentionnent rien car l'accès à ces usuels nest possible que depuis l'ENT ou depuis les postes informatiques au sein de l'université (c'est le cas pour le SCD de Nancy 2 par exemple).
Tableau 5.

Types de documents numériques proposés sur les sites de SCD

\begin{tabular}{|c|c|c|}
\hline $\begin{array}{l}\text { RESSOURCES } \\
\text { ÉLECTRONIQUES }\end{array}$ & NOMBRE CITÉ & FréQuence \\
\hline Non réponse & 19 & $21,10 \%$ \\
\hline Base de données & 57 & $63,30 \%$ \\
\hline Périodiques électroniques & 64 & $71,10 \%$ \\
\hline E-books & 18 & $20,00 \%$ \\
\hline Usuels en ligne & 23 & $25,60 \%$ \\
\hline Théses numérisées & 31 & $34,40 \%$ \\
\hline Mémoires numérisés & 8 & $8,90 \%$ \\
\hline Total observé & 90 & \\
\hline
\end{tabular}

Tableau 6.

Autres services proposés sur les sites de SCD

\begin{tabular}{|c|c|c|}
\hline AUTRES SERVICES & NOMBre Cité & FréQuence \\
\hline Non réponse & 13 & $14,40 \%$ \\
\hline $\begin{array}{l}\text { Signets } \\
\text { bibliothèconomiques }\end{array}$ & 37 & $41,10 \%$ \\
\hline Signets thématiques & 27 & $30,00 \%$ \\
\hline Signets régionaux & 9 & $10,00 \%$ \\
\hline Choix des langues & 23 & $25,60 \%$ \\
\hline Demande de PEB en ligne & 40 & $44,40 \%$ \\
\hline $\begin{array}{l}\text { Service de questions } \\
\text { réponses }\end{array}$ & 15 & $16,70 \%$ \\
\hline Didacticiels & 9 & $10,00 \%$ \\
\hline $\begin{array}{l}\text { Liens vers les autres sites } \\
\text { de SCD de la même ville }\end{array}$ & 18 & $20,00 \%$ \\
\hline Total observé & 90 & \\
\hline
\end{tabular}

On peut voir que les thèses numérisées se développent, ce qui est beaucoup moins le cas pour les mémoires. Là encore, certaines universités ou écoles numérisent bien des mémoires, mais n’y donnent accès que depuis un intranet pour des questions de droits d'auteur notamment.

Le Tableau 6 énumère les autres services offerts sur les sites de SCD.

Nous avons choisi de préciser le contenu des signets en les classant en plusieurs catégories, un même site pouvant présenter des signets dans plus d'une de ces catégories. Les signets bibliothéconomiques désignent les liens vers des ressources de type SUDOC7, $\mathrm{BNF}^{8}$, BPI 9 , CADIST ${ }^{10}$, etc. Les signets thématiques

\footnotetext{
7. Service Universitaire de DOCumentation, qui donne notamment accès au catalogue commun de l'ensemble des BUs françaises: www.sudoc.abes.fr/

8. Bibliothèque Nationale de France : www.bnf.fr/

9. Bibliothèque Publique d'Information : www.bpi.fr/

10. Centres d'Acquisition et de Diffusion de l'Information Scientifique et Technique. Créés depuis 1980, ils constituent un réseau documentaire organisé par disciplines de bibliothèques spécialisées au service de la recherche. Ils sont gérés par des BUs ou des établissements de recherche. Pour plus d'informations voir : www.sup.adc. education.fr/bib/intro/Cadist.htm
} 
correspondent à des liens vers des ressources thématiques du ou des domaines de spécialités de l'université de rattachement (Institut Pasteur en médecine, CAIRN en sciences humaines, etc.). Enfin, les signets régionaux sont des liens d'une portée plus limitée géographiquement ("sortir à Nancy», programmes d'institutions culturelles locales par exemple).

Les résultats que nous présentons restent malheureusement incomplets car ils ne tiennent compte du contenu des intranets que dans la mesure où ceux-ci étaient décrits dans les parties du site accessible à tous. Nous navions pas davantage accès aux ENT de ces universités. Néanmoins, cela nous donne une image du contenu des sites des SCD aujourd'hui.

\section{Le site du SCD : un moyen de communication?}

Si les sites Web des SCD sont riches de documentation numérique, il n'en demeure pas moins que cette documentation est souvent fort peu utilisée par les usagers. Nous avons effectué une enquête rapide auprès de 347 étudiants de l'Université de Nancy 2. Ils ne constituent pas une représentation homogène de l'ensemble des filières ou des années détude puisque 54 d'entre eux font partie de filières de communication de la Faculté de lettres $\left(\mathrm{L}_{2}, \mathrm{~L}_{3}, \mathrm{M} 1\right), 141$ sont en première année de DUT (département Informatique, Techniques de Commercialisation, Gestion des Entreprises et des Administrations, Communication d'Entreprise), 103 sont en deuxième année et 22 en licences professionnelles rattachées aux mêmes filières. Il s'agissait avant tout d'avoir quelques indications sur léventuelle opinion des étudiants par rapport au site. En fait, 291 étudiants, soit 83,9\% des étudiants interrogés, ignoraient l'existence du site du $\mathrm{SCD}$. Pourtant cette méconnaissance ne correspond pas à un désintérêt pour la documentation dans son ensemble. Deux cent cinq étudiants $(59,1 \%)$ déclarent emprunter des documents à la bibliothèque et 61 , soit $17,6 \%$, utilisent les ressources électroniques disponibles dans les locaux mêmes des bibliothèques universitaires. La médiation et lincitation des bibliothécaires présents semblent donc avoir un impact important. Si l'on réduit léchantillon aux 32 étudiants qui utilisent le site du SCD, on constate que $75 \%$ d'entre eux empruntent des documents dans les bibliothèques et 49,9\% utilisent les ressources documentaires également à la bibliothèque. Ainsi, il semble que les étudiants qui utilisent le site sont ceux qui, d'une manière générale, sollicitent le plus les ressources documentaires qui leur sont offertes. Une autre observation s'avère intéressante: ce sont les étudiants d'informatique qui utilisent le moins les ressources des bibliothèques puisque seulement $12,8 \%$ d'entre eux empruntent des documents contre $67,9 \%$ à $75 \%$ pour les autres étudiants de l'IUT interrogés en fonction de leur filière d’appartenance. Ils utilisent également moins que les autres les ressources électroniques disponibles à la bibliothèque et le site du SCD. Comme ces étudiants sont aussi censés se procurer de la documentation, on peut penser qu'ils privilégient, plus que les autres et jusquà l'exclusivité pour la grande majorité d'entre eux, ce qu'ils trouvent sur le Web hors site de leur SCD.

Afin d'ouvrir de nouvelles perspectives d'utilisation, on peut envisager un site de SCD comme un moyen de communication. Une communication qui vise des cibles.

Les usagers du SCD forment de multiples cibles internes, composées par les étudiants, les enseignants et les chercheurs ou enseignants/chercheurs. En fait, on peut définir ces cibles internes en fonction des types de recherche suivants :

- étudiants de premier cycle, personnels (enseignants) à la recherche de documents pour ce public ;

- étudiants et personnels effectuant des activités de recherche scientifique.

Nous sommes donc en présence de deux grandes catégories qui ne sont pas homogènes. En effet, si les recherches sont du même genre, il n'en reste pas moins que l'on peut supposer que le personnel, présent plus longtemps dans létablissement que les étudiants, ait une meilleure connaissance des ressources, et que les étudiants de filières professionnalisées aient besoin douvrages plus professionnels que des étudiants de filière générale, etc.

Les publics des deux principales catégories ne sont généralement pas différenciés sur le plan des interfaces bien que leurs attentes et les documents et/ou services susceptibles de les intéresser soient différents. À la première catégorie, on retrouve les bases de données du type grand public telles que Généralis, Delphes, Kompass, Le Monde, etc. ; les périodiques électroniques susceptibles de répondre à leurs besoins (Le Point, L'Express, Historia, Livres Hebdo, LSA, etc.); les mémoires correspondant à leur formation (par exemple, mémoire de stage de DUT). À la deuxième catégorie correspondent les bases de données scientifiques, les bouquets de périodiques scientifiques, les thèses, etc. Bien sûr, il est possible que la première catégorie puisse être intéressée par des ressources plus pointues, mais cela n'est pas souvent le cas. Des interfaces plus adaptées à ces différents publics permettraient une meilleure mise en adéquation des besoins et des moyens d'y répondre.

$\mathrm{Au}$ problème d'interface s'ajoute le fonctionnement actuel des abonnements par bouquet (surtout pour les périodiques électroniques) qui rend difficile, voire impossible, la différenciation des catégories d'usagers. Tout en accroissant considérablement loffre, elle prive aussi les bibliothécaires de leur choix d'acquisition et, par là, d'une forme de contrôle sur ce qui est proposé. Il en va de même avec les e-books, souvent vendus par bouquets eux aussi. On peut regretter que cette innovation s'accompagne ainsi d'une sorte de dépossession des collections qui conduit à une opacité de loffre pour les personnels mais surtout pour les usagers (nombre très important de titres proposés, mauvaises utilisations des interfaces par ces mêmes usagers souvent déroutés). 
Outre les évidentes cibles internes, on peut envisager la prise en compte par le site du SCD de cibles externes : les étudiants et/ou personnel d'autres universités ou certaines personnes intéressées par un sujet. Il va sans dire que la documentation nécessitant un abonnement ne peut alors être accessible. La possibilité d'inscrits extérieurs existe mais elle pose des problèmes de gestion de laccès aux documents numériques à partir de postes extérieurs à l'université. De plus, les cibles externes ne peuvent bénéficier de l'interface proposée via l'ENT puisqu'ils n'y ont pas accès. Pourtant, le public de l'extérieur est susceptible de s'intéresser à la documentation suivante :

- les mémoires de l'université en ligne ;

- un catalogue utilisé comme base de données ;

- des produits éditoriaux émanant des BUs ;

- un service de questions/réponses en ligne.

La mise en ligne avec un accès libre des thèses devrait donner une visibilité accrue à ces documents sous-utilisés; on constate que les liens établis depuis le SUDOC accroissent leur utilisation potentielle. La mise à disposition des mémoires est plus compliquée (cession des droits de la part des auteurs, problèmes techniques de gestion, etc.) et pourrait s'accompagner d'un système de centralisation, thématique par exemple qui en permettrait, à terme, une utilisation nationale, voire internationale. Ce mouvement est en route. Le dépôt des documents universitaires sous format numérique est de plus en plus courant; les thèses font lobjet de ce type de dépôt depuis l'an 2000 au SCD de l'Université Henri Poincaré de Nancy 1, et sont déposées sous forme électronique uniquement depuis 2007, sans qu'il y ait beaucoup de réticences de la part des étudiants-auteurs. Un autre indicateur vient des prêts entre bibliothèques qui diminuent puisqu'il est de plus en plus courant de communiquer une version numérisée des thèses ou mémoires demandés, que cette version existe déjà ou que la numérisation soit faite à la demande, ce qui conduira d'ailleurs au stockage du fichier par la bibliothèque émettrice. C'est le cas actuellement au SCD de l'Université Henri Poincaré de Nancy 1. Les systèmes d'archives ouvertes pourraient être une alternative. Par exemple, actuellement, le site ArchiveSIC ${ }^{11}$ ne recense pas les mémoires. On pourrait imaginer l'intégration de certains mémoires ayant obtenu des notes suffisantes, sur initiative des auteurs par exemple. Bien sûr, cela constituerait une surcharge pour le site.

L'utilisation d'un catalogue comme ressource bibliographique est habituel. Le recours possible au SUDOC rend caduc un intérêt spécifique pour tel ou tel catalogue de BU.

Les produits éditoriaux semblent promis à un avenir intéressant sur la toile. Les utilisateurs sont à la recherche de sources fiables et ergonomiques. Le succès des services offerts par la Bibliothèque municipale de

11. http://archivesic.ccsd.cnrs.fr/
Lyon montre l'intérêt éprouvé par le public. Dans un contexte d'autonomie des universités, on peut penser que la transformation d'un site de SCD en ressource, sur une thématique par exemple, reconnu largement hors de l'université d'origine pourrait constituer un atout. Au-delà de ce point, on peut y voir également la contribution des professionnels des bibliothèques à lenrichissement global du Web et la revalorisation de ces professionnelles au sein de la nouvelle donne informationnelle. Des initiatives existent en ce sens telles que la "jubilothèque », un portail documentaire de l'UPMC (Université Pierre et Marie Curie à Paris) qui propose des dossiers documentaires en ligne en fonction de l'actualité de l'Université. Par exemple, dans le cadre de l'exposition "Orages! Ô rage!», un dossier contenant une bibliographie et une webographie est mis en ligne. Vingt dossiers sont actuellement disponibles ${ }^{12}$. Cette même bibliothèque propose une bibliothèque numérique composée douvrages anciens numérisés. Leur bon recensement, notamment par les moteurs de recherche, est alors indispensable.

Les services de questions/réponses en ligne vont dans le même sens. Différents SCD offrent déjà ce service, qui, s'il vise en premier lieu les usagers du SCD, peut sétendre plus largement aux internautes qui en font la demande ${ }^{13}$. Dans d'autres pays, une centralisation a été mise en place. Il est probable qu'une telle organisation devrait pouvoir émerger en France. Pour les bibliothèques danoises par exemple, il s'agit d'une capitalisation de l'ensemble des ressources des bibliothèques publiques (catalogue et prêt "automatique » entre bibliothèques, services questions/réponses, etc.), qui inclut une centralisation des services de questions/réponses (Hapel, 2006). Le SCD de l'Université Henri Poincaré de Nancy 1 propose ce service depuis plus d'un an. Il reçoit, en moyenne, une question par jour. En fait, deux voies sont possibles : passer par l'ENT et faire un «ticket", ou passer par le site de la bibliothèque et remplir une demande. Les questions venant de l'ENT sont plus techniques (problèmes de connections, d'accès) que celles qui ont été posées via le site de la bibliothèque. Ces dernières sont davantage tournées vers des points touchant aux collections et à leur utilisation.

\section{Difficultés et contraintes}

Avant de mettre en place de tels services, il faut résoudre d'autres types de difficultés. Le problème des accès multiples se pose aujourd'hui aux SCD. Or, compte-tenu de ces différentes utilisations et des moyens disponibles, il paraît important de réussir à articuler les services, les offres et les lieux virtuels d'accès. La présence des SCD sur les ENT est une obligation. De même, l'existence d'une ou plusieurs pages du SCD sur

\footnotetext{
12. Voir sur le site Jubilothèque : http://jubil.upmc.fr/

13. Voir par exemple le site du SCD de l'Université Henri Poincaré de Nancy 1 : www. scd-uhp.fr/
} 
le site de l'université semble évidente. Un site autonome auquel aboutit directement une requête sur le Web est également utile. Il faut alors déterminer ce que doivent contenir ces différents sites : doivent-ils être redondants, tout en bloquant l'accès aux personnes extérieures pour les ressources payantes? Doivent-ils contenir des documents et services différents? Deux de ces sites doiventils renvoyer vers LE site du SCD? La réponse à cette question dépasse le point de vue ergonomique et renvoie à (aux) identité(s) même du SCD, à l'image qu'il veut projeter de lui-même, aux cibles auxquelles s'adresse chacune de ses applications.

Les responsables du site du SCD de l'Université Henri Poincaré de Nancy 1 ont depuis longtemps mis en place un site Web14. Celui-ci est particulièrement bien recensé par les moteurs de recherche. On a comptabilisé 169682 visites en $2007,70 \%$ d'entre elles ayant cependant duré moins de 10 secondes. Néanmoins, sur les 64649 internautes différents qui ont visité le site, $63 \%$ sont venus plus d'une fois. Hormis la page d'accueil, le service le plus visité concerne les bases de données, suivi par les revues en ligne, les catalogues, les sélections de ressources Web (qui ne sont pourtant plus mises à jour), les configurations d'accès, les encyclopédies et dictionnaires en ligne, la documentation numérique, la page des renseignements en ligne. Les responsables du SCD ont fait le choix de ne proposer qu'un seul site à leurs lecteurs : le site disponible sur le Web et le site disponible via l'ENT sont donc un seul et même site dont seules les possibilités d'accès diffèrent. En 2007, $49 \%$ des visiteurs ont accédé au site directement, $19 \%$ via Google, $15 \%$ via l'ENT, et $9 \%$ via des sites de l'université15.

Bien que lon puisse estimer que ces données sont particulièrement satisfaisantes, les responsables du SCD ont le désir d'animer et de faire vivre leur site afin d'en améliorer encore les services et la fréquentation, notamment en proposant davantage d'actualités et d'informations ponctuelles. Cela les oblige à prendre en compte un certain nombre de contraintes liées à la gestion informatique, laquelle est confiée à un service universitaire commun. De ce fait, ils ne peuvent pas proposer certaines des applications permises par le Web 2.0 telles que des fils RSS. Afin de compenser une certaine forme d'immobilisme, ils ont mis en ligne un blog proposant des sélections de documents et des fiches de lecture. Lidée est également d'inciter les usagers à contribuer au contenu des sites. Cela vise les enseignants bien sûr, dont l'expertise sur la qualité des ouvrages serait particulièrement intéressante, mais aussi les étudiants. Les ouvrages présentés connaissent effectivement un succès indéniable. Dans ce cas également, il est nécessaire de coordonner les informations données en ligne à la réalité physique des lieux : si un ouvrage fait lobjet d'une mise

14. Merci à Jean-Charles Houpier et à Jean-Christophe Brochard, conservateurs au SCD de l'Université Henri Poincaré de Nancy 1, pour les données qu'ils m'ont communiquées ainsi que pour leur disponibilité et leur chaleureux accueil.

15. L'ensemble de ces données provient d'une source interne du SCD. en avant sur le blog, il doit être facilement repérable au sein de la bibliothèque.

Enfin, l'harmonisation de l'ensemble des ressources documentaires est également à prendre en compte dans une logique plus globale touchant à l'ensemble de l'université. À l'heure actuelle, les articles des chercheurs de l'université ne sont pas encore stockés par le SCD. De même, les documents pédagogiques sont gérés par le service des TICE et non par le SCD. Plusieurs accès sont donc nécessaires et plusieurs thésaurus coexistent.

Face aux enjeux actuels d'une dématérialisation toujours plus importante, il paraît indispensable de rendre les opérations de plus en plus transparentes pour l'usager : la mise en lien des fiches bibliographiques trouvées dans une base de données avec les articles en texte intégral présents dans des périodiques en ligne auxquels la bibliothèque est abonnée permet, par exemple, une suppression des démarches de localisation des documents, fort appréciée des lecteurs. Les innovations techniques telles que les résolveurs de liens permettent de simplifier la mise à jour pour ce type de services. La mise en ligne des thèses et des mémoires participe de la même simplification. L’offre éditoriale numérique est de plus en plus importante et intéressante; elle permet la recherche en texte intégral et donne accès à un nombre toujours plus important d'ouvrages et d'usuels sur un support unique et depuis des lieux variés. Les évolutions techniques, comme celles liées à la gestion même des structures, sont incessantes. Toutes les SCD des universités nancéiennes devraient fusionner à l'horizon 2012. Il s'agira alors de relever ce nouveau défi.

\section{Conclusion}

Les sites Web des bibliothèques sont passés du stade de simple reflet des lieux physiques à une existence et une fonction qui leur soient propres. Les avancées technologiques permettent de plus en plus leur utilisation en lieu et place des bibliothèques physiques. Ce mouvement n'ira quen s'accentuant avec le dépôt des thèses et mémoires en format numérique et lémergence annoncée des e-books. Néanmoins, il y a souvent un décalage entre la richesse et la technicité de ce qui est proposé et les pratiques des utilisateurs lesquels, comme la montré notre enquête pour ce qui est des premiers cycles tout au moins, continuent à privilégier une présence sur place, les documents imprimés et les conseils des bibliothécaires. L'évolution des sites des SCD n’est pas terminée. En plus des innovations techniques, gageons que les années qui viennent verront également émerger de nouveaux services, voire une évolution du métier de bibliothécaire en BU de moins en moins pris par les tâches bibliothéconomiques traditionnelles (acquisitions, catalogage, indexation, rangement...) et de plus en plus tourné vers un rôle de créateur d'interfaces, mais aussi de produits éditoriaux, de documents secondaires dont le besoin est de plus en plus prégnant. Il est nécessaire « d’amener les 
documents là où sont les lecteurs $»^{16}$ et de donner accès depuis le Web à l'intégralité de la documentation. Mais aussi et surtout, de faire savoir la richesse des fonds et des services, d'expliquer, au-delà des simplifications techniques, comment, et même parfois, pourquoi utiliser les ressources proposées. Les bibliothécaires ont su sadapter aux changements techniques profonds qui ont touché leur profession depuis une dizaine d'années, aujourd'hui l'enjeu semble de plus en plus se situer au niveau de la mise en place de réelles stratégies de communication. $(-$

\section{Sources consultées}

Association des bibliothécaires de France. 2007. Le métier de bibliothécaire. Sous la direction de Raphaëlle Mouren et de Dominique Peignet. Paris : Electre / Cercle de la Librairie.

Bourrion, Daniel. 2007. Se fondre dans le grand tout : l'intégration du SCD dans l'environnement numérique de travail. Bulletin des bibliothèques de France. 52 (6) : 34-38.

Hapel, Rolf. 2006. Transformer les bibliothèques : la stratégie danoise pour la société de l'information. Bulletin des bibliothèques de France. 51 (3): 64-67.

Jubilothèque. $<$ http://jubil.upmc.fr $>$ (consulté sur Internet le 27 mars 2008).

Service Commun de Documentation de l'Université Nancy 2. $<$ http://scd.univ-nancy2.fr>(consulté sur Internet le 3 juin 2007).

Service Commun de Documentation de l'Université Henri Poincaré de Nancy 1. <http://scd.uhp-nancy.fr> (consulté sur Internet le 3 juin 2007). 